\title{
Suspected Bacterial Endophthalmitis Following Sustained-release Dexamethasone Intravitreal Implant: A Case Report
}

\author{
Mücella Arıkan Yorgun ${ }^{1}$, Melek Mutlu, Yasin Toklu, Hasan Basri Çakmak², Nurullah Çağıl ${ }^{2}$ \\ ${ }^{I}$ Ophthalmology Department, Ankara Atatürk Training and Research Hospital, Ankara, Turkey \\ ${ }^{2}$ Department of Ophthalmology, Ylldirım Beyazıt University, Ankara, Turkey
}

\begin{abstract}
A 58-year-old man admitted to our opthalmology department with the complaint of branch retinal vein occlusion. He was treated with intravitreal Ozurdex in the right eye. Two days after the injection, the patient presented with ocular pain and the visual acuity was hand movement. A diagnosis of endophthalmitis was made. We performed emergent pars plana vitrectomy (PPV) and the implant was removed from the vitreous cavity using a retinal forceps. A combination of vancomycin $1.0 \mathrm{mg}$ and amikacin $0.4 \mathrm{mg}$ was injected intravitreally. However, because of the blurring in the vitreus one week after the procedure, phacoemulsification and a repeat PPV was performed. Five days after the last procedure the signs and symptoms of endophthalmitis were resolved. Our case demonstrated that endophthalmitis could develop after intravitreal implantation of Ozurdex. Surgical removal of the implant and immediate vitrectomy seems to be a useful treatment option in these cases.
\end{abstract}

Key Words: Dexamethasone implant, Endophthalmitis, Ozurdex, Pars plana vitrectomy

Intravitreal therapy has been consistently used for macular edema following branch retinal vein occlusion (BRVO) and central retinal vein occlusion (CRVO). Recent advances in intravitreal implant technology have led to the use of sustained-release dexamethasone intravitreal implant in cases of BRVO [1]. In the larger studies, including the ZERO Study and the Ozurdex GENEVA Study Group, which evaluated the use of intravitreal dexamethasone (Ozurdex; Allergan, Irvine, CA, USA) for the treatment of macular edema due to retinal vein occlusion, the authors reported favorable results without major complications including infections or endophthalmitis [1,2].

To the best of our knowledge, there is limited data regarding the incidence of acute endophthalmitis after sus-

Received: February 4, 2014 Accepted: April 7, 2014

Corresponding Author: Mücella Arıkan Yorgun, MD. Ophthalmology Department, Ankara Atatürk Training and Research Hospital, Bilkent, Ankara, Turkey. Tel: 90-505-351-7118, Fax: 90-312-291-2525, E-mail: mcllarkn@yahoo.com tained-release dexamethasone intravitreal implant [3]. In this manuscript, we aimed to report a rare case of a patient who presented with acute endophthalmitis following sustained-release dexamethasone intravitreal implant for BRVO.

\section{Case Report}

A 58-year-old man was admitted to our opthalmology department with the complaint of decreased visual acuity. The past medical history was unremarkable for any systemic illness or drug use. Best-corrected visual acuity (BCVA) was 0.2 on Snellen's acuity chart in the right eye, 1.0 in the left eye and bilateral intraocular pressure meaurements were in normal limits. Fundus fluorescein angiography revealed non-ischemic BRVO and macular edema in the right eye. Optical coherance tomography revealed cystoid macular edema with a central retinal thickness of $430 \mu \mathrm{m}$. The patient underwent intravitreal ranibizumab injection afterwards; however there was not any improve- 
ment in macular edema. As a result, we decided to implant intravitreal sustained-release dexamethasone (Ozurdex) to the patient with cystoid macular edema because of CRVO.

The procedure was performed in the operating room adhering strictly to the rules of sterilization. The physician and all other medical staff wore caps and surgical masks. We used a single-use sterile injection set and the instruments were prepared on a sterile tray. A sterile anesthetic drop was placed in the appropriate eye. A periocular scrub using $10 \%$ povidone iodine (PVI) was performed, and $5 \%$ PVI drop was placed on the ocular surface before the injection. A fenestrated self-adhesive surgical drape that covered the patient's nose and mouth, and a sterile lid speculum were used. Following the injection, the eye was closed with sterile compresses. Lomefloksasin eye drops were prescribed twice daily for 3 days after the procedure.

Two days after the procedure, the patient presented with ocular pain, blurred vision and photophobia. The visual acuity was limited to hand movement in the right eye. Significant anterior chamber reaction with hypopyon, moderate corneal edema and severe vitritis were detected on examination (Fig. 1). The red reflex was absent in the fundus examination. B-scan ultrasonography showed vitreous opacification, but no retinal detachment.

According to the results described above, a diagnosis of endophthalmitis was made. We subsequently performed emergent 23-gauge pars plana vitrectomy (PPV) and the implant was removed from the vitreous cavity using a retinal forceps (Fig. 2). Aqueous and vitreus sampling for microbiological analysis was taken. In the same session, a combination of vancomycin $1.0 \mathrm{mg}$ and amikacin $0.4 \mathrm{mg}$ was injected intravitreally. The same intravitreal antibiotic regimen was repeated 2 days after the intervention. We diagnosed cataract formation one week after the procedure and the blurring in the vitreus was still present. Phacoemulsification and a repeat PPV was performed. Five days after the last procedure, the signs and symptoms of endophthalmitis were resolved with total remission of pain and hypopyon. The final BCVA was 0.05 for the right eye. The results of both aqueous and vitreous cultures were negative.

\section{Discussion}

Endophthalmitis is one of the most feared complications of ophthalmic invasive procedures, and appears to be a

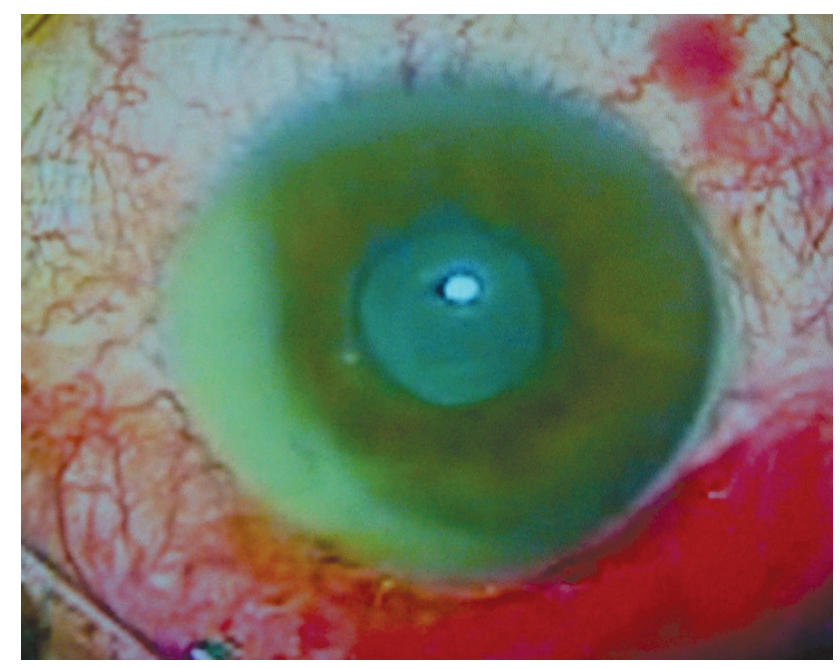

Fig. 1. Significant anterior chamber reaction with hypopyon.

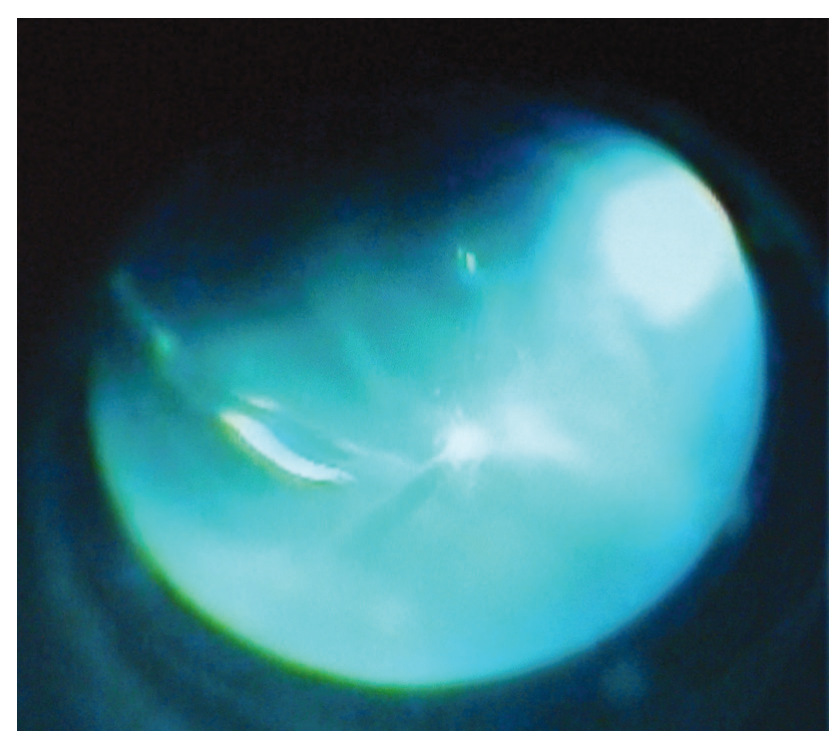

Fig. 2. The implant was removed from the vitreous cavity using a retinal forceps.

very rare complication of sustained-release dexamethasone intravitreal implant.

Ozurdex is a type of intravitreal therapy which is designed to provide sustained delivery of $700 \mu \mathrm{g}$ of preservative-free dexamethasone to the vitreous cavity and retina. Ozurdex has been recently approved by the United States Food and Drug Administration for the treatment of macular edema associated with CRVO and BRVO [4]. Several clinical studies revealed favorable results regarding the beneficial effects of Ozurdex in the treatment of BRVO $[1,2]$. In addition, researchers reported limited side effects 
without any infection or endophthalmitis, but pain, ocular hypertension, anterior chamber cells, cataracts, vitreous hemorrhage, and retinal detachment did occur.

Despite the attempts to provide aseptic conditions before injection in order to avoid microbial contamination causing acute endophthalmitis, the patient developed complications involving endophthalmitis 2 days after Ozurdex implantation. Therefore, because of the loss in vision to hand motion, we immediately performed PPV and removed the implant on the same day as the intravitreal antibiotic injection. Unlike endophthalmitis after cataract extraction for which specific guidelines were developed by the Endophthalmitis Vitrectomy Study [5], there is no established treatment protocol for endophthalmitis after intravitreal implantation of Ozurdex. Although our patient was treated with intravitreal antibiotics and PPV on the same day of presentation, we did not follow a strict treatment protocol for endophthalmitis regarding indications for vitrectomy surgery. Similarly, previous reports recommended immediate vitrectomy to reduce the bacterial overload and improve the outcome before the loss of vision according to the prespecified criteria defined by the Endophthalmitis Vitrectomy Study [6].

We think that surgical removal of the implant and immediate PPV is an important step in the management of endophthalmitis. Given the increasing number of injections in clinical practice, the overall number of endophthalmitis cases is expected to increase as well. Therefore, there seems to be an emerging need to make a consensus statement for endophthalmitis regarding the intravitreal implant procedure.

Despite the unique characteristics seen in this case report, we are aware of some limitations associated with this case including the culture negativity and the nonmethodical treatment algorithm that we followed. The culture was negative in our case; therefore we can not exclude the possibilty that noninfectious injection-related inflammation might be mistakenly attributed to infectious endophthalmitis. In cases of identified bacterial strains, it is helpful to select the appropriate antibiotics for treatment. However, the issue of empirical treatment with antibiotics covering potential microorganisms should be kept in mind, even if the culture is negative.

In conclusion, our case demonstrated that endophthalmitis could develop after intravitreal implantation of Ozurdex. Surgical removal of the implant and immediate vitrectomy seems to be a useful treatment option in these cases. Further studies are needed to establish a treatment algorithm for the treatment of endophthalmitis after sustained-release dexamethasone intravitreal implant.

\section{Conflict of Interest}

No potential conflict of interest relevant to this article was reported.

\section{References}

1. Schmitz K, Maier M, Clemens CR, et al. Reliability and safety of intravitreal Ozurdex injections. The ZERO study. Ophthalmologe 2014;111:44-52.

2. Haller JA, Bandello F, Belfort R Jr, et al. Dexamethasone intravitreal implant in patients with macular edema related to branch or central retinal vein occlusion twelve-month study results. Ophthalmology 2011;118:2453-60.

3. Marchino T, Vela JI, Bassaganyas F, et al. Acute-onset endophthalmitis caused by alloiococcus otitidis following a dexamethasone intravitreal implant. Case Rep Ophthalmol 2013;4:37-41.

4. London NJ, Chiang A, Haller JA. The dexamethasone drug delivery system: indications and evidence. Adv Ther 2011; 28:351-66.

5. Results of the Endophthalmitis Vitrectomy Study. A randomized trial of immediate vitrectomy and of intravenous antibiotics for the treatment of postoperative bacterial endophthalmitis. Endophthalmitis Vitrectomy Study Group. Arch Ophthalmol 1995;113:1479-96.

6. Kuhn F, Gini G. Ten years after... are findings of the Endophthalmitis Vitrectomy Study still relevant today? Graefes Arch Clin Exp Ophthalmol 2005;243:1197-9. 